\title{
From limbs to leaves: common themes in evolutionary diversification of organ form
}

\author{
Remco A. Mentink and Miltos Tsiantis* \\ Department of Comparative Development and Genetics, Max Planck Institute for Plant Breeding Research, Cologne, Germany
}

An open problem in biology is to derive general principles that capture how morphogenesis evolved to generate diverse forms in different organisms. Here we discuss recent work investigating the morphogenetic basis for digit loss in vertebrate limbs and variation in form of marginal outgrowths of angiosperm (flowering plant) leaves. Two pathways underlie digit loss in vertebrate limbs. First, alterations to digit patterning arise through modification of expression of the Patched 1 receptor, which senses the Sonic Hedgehog morphogen and limits its mobility in the limb bud.

OPEN ACCESS

Edited by:

Sylvain Marcellini,

University of Concepcion, Chile

Reviewed by:

Barbara Ambrose,

The New York Botanical Garden, USA

Cédric Finet,

University of Cambridge, UK

*Correspondence:

Miltos Tsiantis,

Department of Comparative

Development and Genetics, Max

Planck Institute for Plant Breeding

Research, Carl-von-Linné-Weg 10,

50829 Cologne, Germany

tsiantis@mpipz.mpg.de

Specialty section:

This article was submitted to Evolutionary and Population Genetics,

a section of the journal

Frontiers in Genetics

Received: 16 June 2015

Accepted: 27 August 2015

Published: 08 September 2015

Citation:

Mentink RA and Tsiantis M (2015)

From limbs to leaves: common

themes in evolutionary

diversification of organ form.

Front. Genet. 6:284.

doi: 10.3389/fgene.2015.00284
Second, evolutionary changes to the degree of programmed cell death between digits influence their development after their initiation. Similarly, evolutionary modification of leaf margin outgrowths occurs via two broad pathways. First, species-specific transcription factor expression modulates outgrowth patterning dependent on regulated transport of the hormone auxin. Second, species-specific expression of the newly discovered REDUCED COMPLEXITY homeodomain transcription factor influences growth between individual outgrowths after their initiation. These findings demonstrate that in both plants and animals tinkering with either patterning or post-patterning processes can cause morphological change. They also highlight the considerable flexibility of morphological evolution and indicate that it may be possible to derive broad principles that capture how morphogenesis evolved across complex eukaryotes.

Keywords: evolution and development, leaflet formation, digit formation, patterning versus post-patterning, morphological diversity

A key question in biology is how morphological diversity is generated. Although plants and animals evolved multicellularity independently, within each kingdom conserved gene regulatory networks (hereafter termed networks) control the development of one or more body parts. In this context evolution operates as a "tinkerer," being strongly influenced by the materials currently at hand as well as prior history (Jacob, 1977; Davidson and Erwin, 2006; Pajoro et al., 2014; Sorrells et al., 2015). Consequently, considerable constraints exist on the evolution of new traits (Pires-daSilva and Sommer, 2003; Davidson and Erwin, 2006; Carroll, 2008; Pires and Dolan, 2012) raising the question of how evolutionary changes to networks that control development may circumvent these constraints.

Both theoretical arguments and empirical evidence suggest that regulatory sequence variation has greater potential for the generation of morphological change than coding sequence variation (Stern, 2000; Carroll, 2008). This is likely because regulatory sequences tend to be organized in highly modular cis elements, leading to their mutation having a lower propensity to generate pleiotropic effects that would compromise development (Stern, 2000; Carroll, 2008; Rebeiz et al., 2015). However, to what degree this broad principle manifests itself in different 
evolutionary lineages and how precisely the balance of conservation versus divergence of different networks creates morphological diversity remain open questions (Stern and Orgogozo, 2008, 2009; Rebeiz et al., 2015).

Other than knowing the types of genetic changes underlying the generation of morphological diversity, an understanding of evolution requires determining how, when and where those genetic changes influence morphogenesis. For example, it remains largely unclear if particular stages or aspects of development tend to be preferentially amenable to evolutionary tinkering. Does evolution primarily target developmental processes that are active during early stages of organ development; or are such early stages less favored by evolution, owing to the potential risk of causing pleiotropic effects that will influence later stages of development? Does diversity largely arise through tinkering with later acting developmental programs that fine-tune organ form after the more fundamental patterns have been laid down? Studies on the emergence of novel insect pigmentation patterns in closely related species suggest that later developmental stages (e.g., the insect pupal stage) might be more readily available for evolutionary tinkering (Wittkopp and Beldade, 2009). However, definitive answers to these questions are likely still to come and will depend on the particularities of the system that is under investigation, including its evolutionary history, its modularity, the type of trait being studied and its degree of integration with other traits. Nevertheless, one way to approach these problems in a unified fashion when comparing diverse organisms is to consider whether and how evolution influences patterning and post-patterning modes of development. Patterning processes act to impart positional information, for example through the use of morphogen concentration gradients, and facilitate correct distribution of cellular identities within tissues (Kondo and Miura, 2010; Rogers and Schier, 2011). Post-patterning processes, on the other hand, serve to sculpt emerging tissues and organs typically after their identity has previously been determined. For example, post-patterning processes may operate by removing superfluous cells through apoptosis or by adjusting the growth rates of specific populations of cells within the organ (Coen et al., 2004; Suzanne and Steller, 2013). Notably, this distinction between "post-patterning" and patterning does not exclude the possibility that patterning genes may have persistent effects in developmental time, including post-patterning stages (SalazarCiudad et al., 2003; McGregor et al., 2007; Werner et al., 2010).

Two recent papers have explored the significance of patterning versus post-patterning events on development by studying digit loss in mammals and leaf shape formation in angiosperms and revealed a strong link between altered, species-specific gene expression domains and morphological variation. Both studies suggest considerable versatility in how evolutionary tinkering with developmental processes can ultimately arrive at similar phenotypes.

Cooper et al. (2014) studied the evolutionary changes that resulted in convergent digit loss in different mammalian species. A mammalian limb (such as a leg) is attached to the body at one (proximal) end and has 1 to 5 anteroposteriorly distinct digits (e.g., toes) at the other (distal) end. Limbs develop from the limb bud through the sequential action of several distinct signaling centers
(Figure 1A; Butterfield et al., 2010). Bone morphogenetic proteins (BMPs) specify the formation of the apical ectodermal ridge (AER) at the distal end of the limb bud, from which fibroblast growth factors (FGFs) are secreted to stimulate proximodistal outgrowth (Lewandoski et al., 2000; Pizette et al., 2001; Boulet et al., 2004). The morphogen Sonic hedgehog (SHH) is secreted from the posterior limb bud to direct both digit patterning and expansion of the hand- or footplate to accommodate all digits (Harfe et al., 2004; Towers et al., 2008). Subsequent digit elongation is controlled by FGFs secreted from the AER and in later stages BMPs sculpt the limb by inducing apoptotic cell death within interdigital tissue in concert with the transcription factor Msx2 (Marazzi et al., 1997; Ferrari et al., 1998; Sanz-Ezquerro and Tickle, 2003).

Cooper et al. (2014) studied the possible relevance of these pathways to digit loss in 3- and 5-toed jerboas (small, desertdwelling rodents that develop a varying number of digits on the hind limb between different species) and mice, as well as in ungulates (hoofed animals with 1 to 4 toes). In jerboas, they observed no differences in patterning gene expression but rather found expanded domains of apoptotic cells in 3-toed jerboa hind limbs, surrounding tissue otherwise destined to form digits I and V. Specifically, they found that expression of Msx2 was expanded in the 3-toed jerboa hind limb, likely causing increased cell death (Figure 1A). They obtained comparable results with 1-toed horse embryos, where Msx2 expansion correlated with removal of digits II and IV. This shows a convergent evolutionary event in which an apoptotic pathway normally used to remove interdigital tissue was co-opted by regulatory changes to act in truncating digit outgrowth.

By expanding their study to even-toed ungulate species, Cooper et al. (2014) found a striking flexibility in modes of digit loss. In pigs the expression of Patched 1 (Ptch1), a Shh receptor, is reduced toward the posterior limb bud. Ptch1 acts to restrict the spread of $S h h$ by sequestration, thus reduction in Ptch 1 expression leads to an expanded region of Shh activity and more uniform expression of its target genes, presumably causing a shift in limb axis symmetry to the space between digits III and IV (Figure 1A; Chen and Struhl, 1996; Butterfield et al., 2009). These findings were corroborated by a second group that showed a similar reduction of Ptch1 expression in cow limb buds (Lopez-Rios et al., 2014). These authors also demonstrated that cis regulatory divergence of Ptch 1 renders it unresponsive to Shh signaling in a negative feedback loop. Remarkably, when Cooper et al. (2014) examined embryos of camel, a third ungulate, they observed no modification of Ptch1 expression, but instead an expansion of apoptosis and Msx2 expression, resembling the case in 3toed jerboas and horses (Figure 1A; Cooper et al., 2014). These results indicate that in species of the same taxonomic order, such as camels and pigs (both members of the Artiodactyla or even-toed ungulates), fundamentally different mechanisms can be modified to achieve similar organ modifications, revealing considerable flexibility in evolutionary pathways. A conclusion that is additionally in line with the fact that Cooper et al. (2014) did not recover any evidence for evolutionary tinkering with the HoxD regulatory landscape, which has previously been identified and hypothesized to be a good candidate for vertebrate digit 


\section{A}
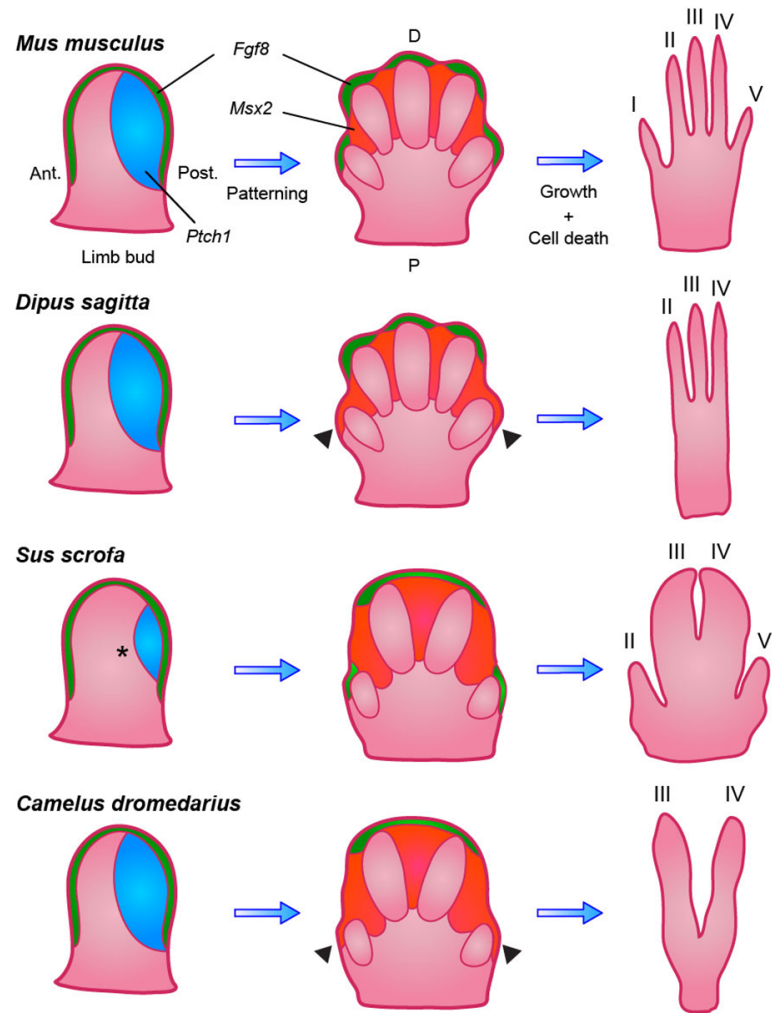
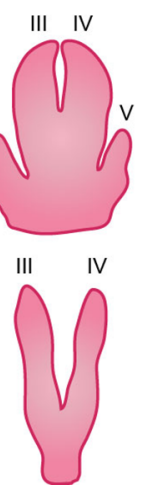

B Arabidopsis thaliana

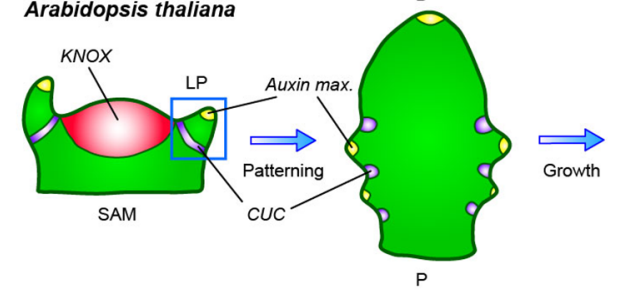

Cardamine hirsuta

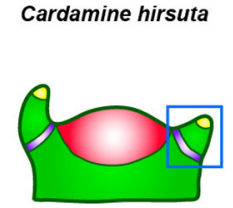

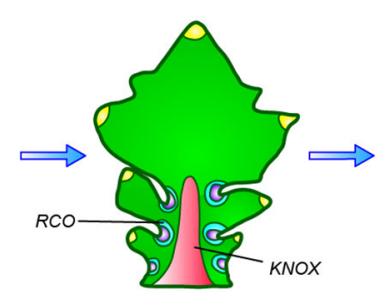
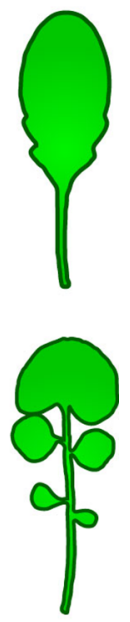

FIGURE 1 | Diversification of both patterning and post-patterning processes contributed to morphological variation of leaves and limbs. (A) In the vertebrate limb bud Fgf8 (green) expression from the apical ectodermal ridge (AER) stimulates proximodistal outgrowth, while posteriorly expressed Ptch1 (blue), through sequestration, creates a posterior to anterior $\mathrm{SHH}$ gradient that patterns the individual digits. In later stages, Fgf8 is maintained only in the AER, overlying digits that will completely form, while Msx2 (orange) expression is turned on in the interdigital tissue, triggering apoptosis. In the 3-toed jerboa and camel, expanded Msx2 expression causes the regression of the outermost digits (indicated by arrowheads). In the pig, Ptch1 expression is reduced to eliminate digit I (indicated by a star), a change that is not observed in the closely related camel. Ant, anterior; Post, posterior; P, proximal; D, distal. (B) Simple and dissected leaves similarly initiate as small leaf primordia (LP) from the shoot apical meristem (SAM) at sites where auxin maxima (yellow) are defined by CUC (purple) and PIN1 expression, but only dissected leaves reactivate KNOX (red) expression to suppress leaf cell differentiation. This allows the initial marginal outgrowths, patterned by CUC and PIN1, to develop into leaflets instead of serrations. Species-specific local expression of RCO (b/ue-green) in Cardamine hirsuta restricts cellular growth within leaf sinuses, thereby allowing separation of individual leaflets through modulation of local growth rates. P, proximal; D, distal.

diversification, owing to its highly modular nature (Montavon et al., 2011). These findings raise the question of how broadly this flexibility in evolutionary tinkering with either growth or patterning occurred during evolution of complex eukaryotes.

A recent paper by Vlad et al. (2014) establishes that a comparable logic helps explain diversification of leaf shapes in plants of the Brassicaceae family. Brassicaceae, like other flowering plants, form either simple leaves, consisting of an entire blade with smooth, serrated or lobed margins, or dissected leaves, comprising individual leaflets. Both types of leaves develop from leaf primordia that initiate from the pluripotent shoot apical meristem (SAM). KNOTTED-LIKE HOMEOBOX (KNOX) transcription factors are expressed in the meristem to maintain its organ-generating potential (Hay and Tsiantis, 2006; Barkoulas et al., 2007). Transport of the plant hormone auxin through the PIN-FORMED 1 (PIN1) efflux transporter, coupled to a self-reinforcing feedback of auxin on PIN1 expression and polarization, likely creates sequential local auxin activity maxima at the flanks of the SAM. This process appears to be self-organizing and the resulting auxin maxima are required for sequential primordium development (Reinhardt et al., 2003; Heisler et al., 2005; Jonsson et al., 2006; Smith et al., 2006). CUP-SHAPED COTYLEDON (CUC) genes mark the leaf primordium boundary and allow its separation by repressing growth at the flanks (Aida et al., 1997; Hibara et al., 2006). CUCs and PIN1 also function together to pattern the leaf margin, as CUCs likely repress growth at the boundaries of serrations or leaflets, while PIN1 generates auxin maxima at the sites of their outgrowth. Notably, in this context CUCs likely both repress growth at the flanks of marginal outgrowths and promote their outgrowth at least in part via promoting generation of an auxin maximum at their tip (Nikovics et al., 2006; Barkoulas et al., 2008; Blein et al., 2008; Koenig et al., 2009; Kawamura et al., 2010; BenGera et al., 2012). In the Arabidopsis thaliana leaf margin, CUC2 directs PIN1 localization to form local auxin maxima while auxin feeds back to repress $C U C 2$, creating the repeated pattern of leaf serrations along the leaf margin (Bilsborough et al., 2011). KNOX genes, then, are expressed in dissected leaves and differentiate these from simple leaves by retarding cellular differentiation, thus rendering the leaf competent to form leaflets in response to PIN1 
dependent auxin maxima (Hay and Tsiantis, 2006; Barkoulas et al., 2008; Kimura et al., 2008; Bar and Ori, 2014). Similarly CUC1, a redundantly acting paralogue of $C U C 2$, is expressed in the dissected leaves of Cardamine hirsuta but is confined to the leaf meristem boundary in its simple-leaved relative $A$. thaliana. These observations indicate that evolutionary tinkering with auxinbased patterning mechanisms through alterations in expression of upstream transcription factors such as KNOX and CUC may be a major route for generating diversity in leaf shapes (Figure 1B; Barkoulas et al., 2008; Blein et al., 2008; Piazza et al., 2010; Hasson et al., 2011; Finet and Jaillais, 2012; Bar and Ori, 2014).

Until recently, no genes had been identified that specifically influence leaflet formation without also affecting meristem function or leaf initiation. Such findings suggested that leaflets form through the redeployment of processes that acted earlier in development during leaf initiation (Bar and Ori, 2014; Vlad et al., 2014). To identify novel regulators of leaf complexity, Vlad et al. (2014) conducted a forward genetic screen for genes required for leaflet formation in $C$. hirsuta. They identified the REDUCED COMPLEXITY (RCO) homeobox gene, of which a loss of function allele simplifies the leaf without causing pleiotropic phenotypes, suggesting a specific requirement for $R C O$ in leaflet formation. $R C O$ evolved in the Brassicaceae family from a gene duplication of LATE MERISTEM IDENTITY 1 (LMI1); originally identified in A. thaliana as a floral regulator (Saddic et al., 2006). They found that $R C O$ is specifically expressed at the base of leaflets (Figure 1B), while LMI1 is expressed more distally, in a complementary pattern, along the leaf margins. $R C O$ does not appear to influence PIN1-mediated auxin patterning, but instead functions by repressing cellular growth between individual leaflets in C. hirsuta, a post-patterning process that allows leaflet separation. $R C O$ was lost in A. thaliana during evolution, contributing to its leaf simplification, but re-introducing $R C O$ into $A$. thaliana drives expression in basal regions of the leaf and increases leaf complexity, partially reversing the consequences of evolution. These results, together with a follow-up study in the sister species Capsella rubella and Capsella grandiflora by Sicard et al. (2014), suggest that $R C O$ is a key regulator of leaf shape and diversity in the Brassicaceae and provide a striking example of organ shape diversification by tinkering with local growth regulation at the flanks of a growing organ primordium (Vlad et al., 2014). Another notable aspect of the $R C O$ study is that this gene was discovered through performing a forward genetics study in C. hirsuta and could not have been found in A. thaliana, where the gene has been lost, thus highlighting the importance of unbiased studies in diverse taxa for understanding the genetic basis for the evolution of form.

Taken together, these two studies illustrate how evolution can exploit both patterning and post-patterning processes to

\section{References}

Abzhanov, A., Protas, M., Grant, B. R., Grant, P. R., and Tabin, C. J. (2004). Bmp4 and morphological variation of beaks in Darwin's finches. Science 305, 1462-1465. doi: 10.1126/science.1098095

Aida, M., Ishida, T., Fukaki, H., Fujisawa, H., and Tasaka, M. (1997). Genes involved in organ separation in Arabidopsis: an analysis of the cup-shaped cotyledon mutant. Plant Cell 9, 841-857. doi: 10.1105/tpc.9.6.841 create morphological diversity in both plants and animals. It will be interesting to explore whether bias might exist for variations of either kind or for particular developmental pathways across different kingdoms. For example, plants and animals have evolved distinct biophysical properties and morphogenetic strategies that pose different constraints for evolution. Whereas animal morphogenesis involves the use of large-scale apoptosis and cell migration, these mechanisms are used to more limited extent (Gunawardena, 2008; Fendrych et al., 2014) or not at all respectively in plants. This is because rigid cell walls in plants somewhat complicate the use of both these mechanisms during development: cell walls typically remain after apoptosis, thereby constraining developmental options, while they make cell migration impossible by preventing the sliding of cells alongside each other. These fundamental differences in the cellular underpinnings of development suggest that morphological diversity in plants mostly arises through tinkering with regional growth rates and growth directionality (Coen et al., 2004), consistent with the findings of Vlad et al. (2014) These particularities of plants, however, do not preclude that changes to such growth-related processes can also contribute to the evolution of animal form (Abzhanov et al., 2004; Wu et al., 2004). In any event, independent of the organism studied, morphology is determined by processes that take place at different levels of organization and yield the final form through complex feedback loops of genetic regulation, signaling and tissue growth (Salazar-Ciudad and Jernvall, 2010; Kennaway et al., 2011; Prusinkiewicz and Runions, 2012). Conceptualizing how activity of gene regulatory networks creates organ shape is consequently not solely intuitive. The computer science and developmental biology interface offers a promising path for resolving such problems in a predictive fashion (Lewis, 2008; Green et al., 2010; Prusinkiewicz and Runions, 2012; Sheth et al., 2012). Quantitative investigations of morphogenesis and the genetic basis of its variation in different organismal lineages will allow us to build a general picture of how organ diversity is generated and maintained. Such studies should also help us understand the basis for and limits of predictability of morphological evolution.

\section{Acknowledgments}

Work in the Tsiantis lab on diversification of leaf shape is supported by a Deutsche Forschungsgemeinschaft (DFG) "Adaptomics" grant TS 229/1-1, a DFG Collaborative Research Center SFB 680 grant on Evolutionary Innovations and a core grant from the Max Planck Society. MT also acknowledges support of the Cluster of Excellence on Plant Sciences. We thank Sheila McCormick for comments on the manuscript.

Barkoulas, M., Galinha, C., Grigg, S. P., and Tsiantis, M. (2007). From genes to shape: regulatory interactions in leaf development. Curr. Opin. Plant Biol. 10, 660-666. doi: 10.1016/j.pbi.2007.07.012

Barkoulas, M., Hay, A., Kougioumoutzi, E., and Tsiantis, M. (2008). A developmental framework for dissected leaf formation in the Arabidopsis relative Cardamine hirsuta. Nat. Genet. 40, 1136-1141. doi: 10.1038/ng.189

Bar, M., and Ori, N. (2014). Leaf development and morphogenesis. Development 141, 4219-4230. doi: 10.1242/dev.106195 
Ben-Gera, H., Shwartz, I., Shao, M. R., Shani, E., Estelle, M., and Ori, N. (2012). ENTIRE and GOBLET promote leaflet development in tomato by modulating auxin response. Plant J. 70, 903-915. doi: 10.1111/j.1365-313X.2012. 04939.x

Bilsborough, G. D., Runions, A., Barkoulas, M., Jenkins, H. W., Hasson, A., Galinha, C., et al. (2011). Model for the regulation of Arabidopsis thaliana leaf margin development. Proc. Natl. Acad. Sci. U.S.A. 108, 3424-3429. doi: $10.1073 /$ pnas. 1015162108

Blein, T., Pulido, A., Vialette-Guiraud, A., Nikovics, K., Morin, H., Hay, A., et al. (2008). A conserved molecular framework for compound leaf development. Science 322, 1835-1839. doi: 10.1126/science.1166168

Boulet, A. M., Moon, A. M., Arenkiel, B. R., and Capecchi, M. R. (2004). The roles of Fgf4 and Fgf8 in limb bud initiation and outgrowth. Dev. Biol. 273, 361-372. doi: 10.1016/j.ydbio.2004.06.012

Butterfield, N. C., Mcglinn, E., and Wicking, C. (2010). The molecular regulation of vertebrate limb patterning. Curr. Top. Dev. Biol. 90, 319-341. doi: 10.1016/S0070-2153(10)90009-4

Butterfield, N. C., Metzis, V., Mcglinn, E., Bruce, S. J., Wainwright, B. J., and Wicking, C. (2009). Patched 1 is a crucial determinant of asymmetry and digit number in the vertebrate limb. Development 136, 3515-3524. doi: 10.1242/dev.037507

Carroll, S. B. (2008). Evo-devo and an expanding evolutionary synthesis: a genetic theory of morphological evolution. Cell 134, 25-36. doi: 10.1016/j.cell.2008.06.030

Chen, Y., and Struhl, G. (1996). Dual roles for patched in sequestering and transducing Hedgehog. Cell 87, 553-563. doi: 10.1016/S0092-8674(00)81374-4

Coen, E., Rolland-Lagan, A. G., Matthews, M., Bangham, J. A., and Prusinkiewicz, P. (2004). The genetics of geometry. Proc. Natl. Acad. Sci. U.S.A. 101, 4728-4735. doi: 10.1073/pnas.0306308101

Cooper, K. L., Sears, K. E., Uygur, A., Maier, J., Baczkowski, K. S., Brosnahan, M., et al. (2014). Patterning and post-patterning modes of evolutionary digit loss in mammals. Nature 511, 41-45. doi: 10.1038/nature13496

Davidson, E. H., and Erwin, D. H. (2006). Gene regulatory networks and the evolution of animal body plans. Science 311, 796-800. doi: 10.1126/science. 1113832

Fendrych, M., Van Hautegem, T., Van Durme, M., Olvera-Carrillo, Y., Huysmans, M., Karimi, M., et al. (2014). Programmed cell death controlled by ANAC033/SOMBRERO determines root cap organ size in Arabidopsis. Curr. Biol. 24, 931-940. doi: 10.1016/j.cub.2014.03.025

Ferrari, D., Lichtler, A. C., Pan, Z. Z., Dealy, C. N., Upholt, W. B., and Kosher, R. A. (1998). Ectopic expression of Msx-2 in posterior limb bud mesoderm impairs limb morphogenesis while inducing BMP-4 expression, inhibiting cell proliferation, and promoting apoptosis. Dev. Biol. 197, 12-24. doi: 10.1006/dbio.1998.8880

Finet, C., and Jaillais, Y. (2012). Auxology: when auxin meets plant evo-devo. Dev. Biol. 369, 19-31. doi: 10.1016/j.ydbio.2012.05.039

Green, A. A., Kennaway, J. R., Hanna, A. I., Bangham, J. A., and Coen, E. (2010). Genetic control of organ shape and tissue polarity. PLoS Biol. 8:e1000537. doi: 10.1371/journal.pbio.1000537

Gunawardena, A. H. (2008). Programmed cell death and tissue remodelling in plants. J. Exp. Bot. 59, 445-451. doi: 10.1093/jxb/erm 189

Harfe, B. D., Scherz, P. J., Nissim, S., Tian, H., Mcmahon, A. P., and Tabin, C. J. (2004). Evidence for an expansion-based temporal Shh gradient in specifying vertebrate digit identities. Cell 118, 517-528. doi: 10.1016/j.cell.2004.07.024

Hasson, A., Plessis, A., Blein, T., Adroher, B., Grigg, S., Tsiantis, M., et al. (2011). Evolution and diverse roles of the CUP-SHAPED COTYLEDON genes in Arabidopsis leaf development. Plant Cell 23, 54-68. doi: 10.1105/tpc.110. 081448

Hay, A., and Tsiantis, M. (2006). The genetic basis for differences in leaf form between Arabidopsis thaliana and its wild relative Cardamine hirsuta. Nat. Genet. 38, 942-947. doi: 10.1038/ng1835

Heisler, M. G., Ohno, C., Das, P., Sieber, P., Reddy, G. V., Long, J. A., et al. (2005). Patterns of auxin transport and gene expression during primordium development revealed by live imaging of the Arabidopsis inflorescence meristem. Curr. Biol. 15, 1899-1911. doi: 10.1016/j.cub.2005.09.052

Hibara, K., Karim, M. R., Takada, S., Taoka, K., Furutani, M., Aida, M., et al. (2006). Arabidopsis CUP-SHAPED COTYLEDON3 regulates postembryonic shoot meristem and organ boundary formation. Plant Cell 18, 2946-2957. doi: $10.1105 /$ tpc. 106.045716
Jacob, F. (1977). Evolution and tinkering. Science 196, 1161-1166. doi: 10.1126/science.860134

Jonsson, H., Heisler, M. G., Shapiro, B. E., Meyerowitz, E. M., and Mjolsness, E. (2006). An auxin-driven polarized transport model for phyllotaxis. Proc. Natl. Acad. Sci. U.S.A. 103, 1633-1638. doi: 10.1073/pnas.0509839103

Kawamura, E., Horiguchi, G., and Tsukaya, H. (2010). Mechanisms of leaf tooth formation in Arabidopsis. Plant J. 62, 429-441. doi: 10.1111/j.1365313X.2010.04156.x

Kennaway, R., Coen, E., Green, A., and Bangham, A. (2011). Generation of diverse biological forms through combinatorial interactions between tissue polarity and growth. PLoS Comput. Biol. 7:e1002071. doi: 10.1371/journal.pcbi.1002071

Kimura, S., Koenig, D., Kang, J., Yoong, F. Y., and Sinha, N. (2008). Natural variation in leaf morphology results from mutation of a novel KNOX gene. Curr. Biol. 18, 672-677. doi: 10.1016/j.cub.2008.04.008

Koenig, D., Bayer, E., Kang, J., Kuhlemeier, C., and Sinha, N. (2009). Auxin patterns Solanum lycopersicum leaf morphogenesis. Development 136, 2997-3006. doi: 10.1242/dev.033811

Kondo, S., and Miura, T. (2010). Reaction-diffusion model as a framework for understanding biological pattern formation. Science 329, 1616-1620. doi: $10.1126 /$ science. 1179047

Lewandoski, M., Sun, X., and Martin, G. R. (2000). Fgf8 signalling from the AER is essential for normal limb development. Nat. Genet. 26, 460-463. doi: $10.1038 / 82609$

Lewis, J. (2008). From signals to patterns: space, time, and mathematics in developmental biology. Science 322, 399-403. doi: 10.1126/science.1166154

Lopez-Rios, J., Duchesne, A., Speziale, D., Andrey, G., Peterson, K. A., Germann, P., et al. (2014). Attenuated sensing of SHH by Ptch1 underlies evolution of bovine limbs. Nature 511, 46-51. doi: 10.1038/nature13289

Marazzi, G., Wang, Y., and Sassoon, D. (1997). Msx2 is a transcriptional regulator in the BMP4-mediated programmed cell death pathway. Dev. Biol. 186, 127-138. doi: 10.1006/dbio.1997.8576

McGregor, A. P., Orgogozo, V., Delon, I., Zanet, J., Srinivasan, D. G., Payre, F., et al. (2007). Morphological evolution through multiple cis-regulatory mutations at a single gene. Nature 448, 587-590. doi: 10.1038/nature05988

Montavon, T., Soshnikova, N., Mascrez, B., Joye, E., Thevenet, L., Splinter, E., et al. (2011). A regulatory archipelago controls Hox genes transcription in digits. Cell 147, 1132-1145. doi: 10.1016/j.cell.2011.10.023

Nikovics, K., Blein, T., Peaucelle, A., Ishida, T., Morin, H., Aida, M., et al. (2006). The balance between the MIR164A and CUC2 genes controls leaf margin serration in Arabidopsis. Plant Cell 18, 2929-2945. doi: 10.1105/tpc.106.045617

Pajoro, A., Biewers, S., Dougali, E., Leal Valentim, F., Mendes, M. A., Porri, A., et al. (2014). The (r)evolution of gene regulatory networks controlling Arabidopsis plant reproduction: a two-decade history. J. Exp. Bot. 65, 4731-4745. doi: 10.1093/jxb/eru233

Piazza, P., Bailey, C. D., Cartolano, M., Krieger, J., Cao, J., Ossowski, S., et al. (2010). Arabidopsis thaliana leaf form evolved via loss of KNOX expression in leaves in association with a selective sweep. Curr. Biol. 20, 2223-2228. doi: 10.1016/j.cub.2010.11.037

Pires-daSilva, A., and Sommer, R. J. (2003). The evolution of signalling pathways in animal development. Nat. Rev. Genet. 4, 39-49. doi: 10.1038/nrg977

Pires, N. D., and Dolan, L. (2012). Morphological evolution in land plants: new designs with old genes. Philos. Trans. R. Soc. Lond. B Biol. Sci. 367, 508-518. doi: $10.1098 /$ rstb.2011.0252

Pizette, S., Abate-Shen, C., and Niswander, L. (2001). BMP controls proximodistal outgrowth, via induction of the apical ectodermal ridge, and dorsoventral patterning in the vertebrate limb. Development 128, 4463-4474.

Prusinkiewicz, P., and Runions, A. (2012). Computational models of plant development and form. New Phytol. 193, 549-569. doi: 10.1111/j.14698137.2011.04009.x

Rebeiz, M., Patel, N. H., and Hinman, V. F. (2015). Unraveling the tangled skein: the evolution of transcriptional regulatory networks in development. Annu. Rev. Genomics Hum. Genet. doi: 10.1146/annurev-genom-091212-153423 [Epub ahead of print].

Reinhardt, D., Pesce, E. R., Stieger, P., Mandel, T., Baltensperger, K., Bennett, M., et al. (2003). Regulation of phyllotaxis by polar auxin transport. Nature 426, 255-260. doi: 10.1038/nature02081

Rogers, K. W., and Schier, A. F. (2011). Morphogen gradients: from generation to interpretation. Annu. Rev. Cell Dev. Biol. 27, 377-407. doi: 10.1146/annurevcellbio-092910-154148 
Saddic, L. A., Huvermann, B., Bezhani, S., Su, Y., Winter, C. M., Kwon, C. S., et al. (2006). The LEAFY target LMI1 is a meristem identity regulator and acts together with LEAFY to regulate expression of CAULIFLOWER. Development 133, 1673-1682. doi: 10.1242/dev.02331

Salazar-Ciudad, I., and Jernvall, J. (2010). A computational model of teeth and the developmental origins of morphological variation. Nature 464, 583-586. doi: 10.1038 /nature 08838

Salazar-Ciudad, I., Jernvall, J., and Newman, S. A. (2003). Mechanisms of pattern formation in development and evolution. Development 130, 2027-2037. doi: $10.1242 /$ dev.00425

Sanz-Ezquerro, J. J., and Tickle, C. (2003). Fgf signaling controls the number of phalanges and tip formation in developing digits. Curr. Biol. 13, 1830-1836. doi: 10.1016/j.cub.2003.09.040

Sheth, R., Marcon, L., Bastida, M. F., Junco, M., Quintana, L., Dahn, R., et al. (2012). Hox genes regulate digit patterning by controlling the wavelength of a Turing-type mechanism. Science 338, 1476-1480. doi: 10.1126/science. 1226804

Sicard, A., Thamm, A., Marona, C., Lee, Y. W., Wahl, V., Stinchcombe, J. R., et al. (2014). Repeated evolutionary changes of leaf morphology caused by mutations to a homeobox gene. Curr. Biol. 24, 1880-1886. doi: 10.1016/j.cub.2014.06.061

Smith, R. S., Guyomarc'h, S., Mandel, T., Reinhardt, D., Kuhlemeier, C., and Prusinkiewicz, P. (2006). A plausible model of phyllotaxis. Proc. Natl. Acad. Sci. U.S.A. 103, 1301-1306. doi: 10.1073/pnas.0510457103

Sorrells, T. R., Booth, L. N., Tuch, B. B., and Johnson, A. D. (2015). Intersecting transcription networks constrain gene regulatory evolution. Nature 523, 361-365. doi: 10.1038/nature14613

Stern, D. L. (2000). Evolutionary developmental biology and the problem of variation. Evolution 54, 1079-1091. doi: 10.1111/j.0014-3820.2000.tb00544.x

Stern, D. L., and Orgogozo, V. (2008). The loci of evolution: how predictable is genetic evolution? Evolution 62, 2155-2177. doi: $10.1111 / \mathrm{j} .1558-5646.2008 .00450 . \mathrm{x}$
Stern, D. L., and Orgogozo, V. (2009). Is genetic evolution predictable? Science 323, 746-751. doi: 10.1126/science.1158997

Suzanne, M., and Steller, H. (2013). Shaping organisms with apoptosis. Cell Death. Differ. 20, 669-675. doi: 10.1038/cdd.2013.11

Towers, M., Mahood, R., Yin, Y., and Tickle, C. (2008). Integration of growth and specification in chick wing digit-patterning. Nature 452, 882-886. doi: $10.1038 /$ nature 06718

Vlad, D., Kierzkowski, D., Rast, M. I., Vuolo, F., Dello Ioio, R., Galinha, C., et al. (2014). Leaf shape evolution through duplication, regulatory diversification, and loss of a homeobox gene. Science 343, 780-783. doi: 10.1126/science. 1248384

Werner, T., Koshikawa, S., Williams, T. M., and Carroll, S. B. (2010). Generation of a novel wing colour pattern by the Wingless morphogen. Nature 464, 1143-1148. doi: $10.1038 /$ nature 08896

Wittkopp, P. J., and Beldade, P. (2009). Development and evolution of insect pigmentation: genetic mechanisms and the potential consequences of pleiotropy. Semin. Cell Dev. Biol. 20, 65-71. doi: 10.1016/j.semcdb.2008.10.002

Wu, P., Jiang, T. X., Suksaweang, S., Widelitz, R. B., and Chuong, C. M. (2004). Molecular shaping of the beak. Science 305, 1465-1466. doi: $10.1126 /$ science. 1098109

Conflict of Interest Statement: The authors declare that the research was conducted in the absence of any commercial or financial relationships that could be construed as a potential conflict of interest.

Copyright (C) 2015 Mentink and Tsiantis. This is an open-access article distributed under the terms of the Creative Commons Attribution License (CC BY). The use, distribution or reproduction in other forums is permitted, provided the original author(s) or licensor are credited and that the original publication in this journal is cited, in accordance with accepted academic practice. No use, distribution or reproduction is permitted which does not comply with these terms. 